\title{
Understanding the Coarsening Behaviors of Nanoporous Gold via In situ Heating
}

Ayman Amr El-Zoka ${ }^{1}$, Jane Howe ${ }^{2,3}$, Roger C. Newman ${ }^{1}$, Stas Dogel ${ }^{3}$, Matthew Reynolds ${ }^{4}$, Hooman Hosseinkhannazer ${ }^{4}$ and Doug D. Perovic ${ }^{2}$.

1. Chemical Engineering and Applied Chemistry, University of Toronto, Toronto, Canada.

2. Materials Science and Engineering, University of Toronto, Toronto, Canada.

${ }^{3 .}$ Hitachi High-Technologies Canada Inc., Toronto, Canada.

4. Norcada Inc., Edmonton, Canada.

Preferential dissolution of a less noble metal from a matrix of two or more metals yields a nanoporous, bicontinuous network. A common example is nanoporous gold (NPG), a material showing promise for use as a catalyst [1]. However, a substantial barrier to the wide scale implementation of NPG is the lack of control of surface area instability due to poorly understood coarsening mechanisms.

Coarsening of NPG was observed in previous studies at temperatures as low as $200{ }^{\circ} \mathrm{C}$, which is significantly lower than the melting point of gold [2]. These findings stimulated studies to suggest $\mathrm{Au}$ surface diffusion was the dominant mechanism, at least at the onset of coarsening. Kinetic Monte Carlo simulations predict (111) faceting in the later stages of growth, which drives coarsening to a plateau [3]. One added complexity in the present work is the addition of $4 \% \mathrm{Pt}$ to the precursor alloy (77Ag-19Au- 4Pt, at \%), proven by electrochemical characterization to impede coarsening due to $\mathrm{Pt}$ pinning of $\mathrm{Au}$ adatoms during surface diffusion [4]. In this work, we explore the coarsening of a thin NPG-Pt specimen using in situ transmission electron microscopy, with the goal of observing the structural and chemical changes in the NPG occurring on the surface and through the thickness. Using a Hitachi HF- 3300 environmental TEM/STEM, we carried out in situ heating studies of the $\mathrm{NPG}$, formed by dealloying $\mathrm{AgAuPt}_{4}$, at $440{ }^{\circ} \mathrm{C}$ in vacuum. A Hitachi heating holder with Norcada MEMs-based heating chip were used in this study. This ETEM is equipped with a secondary electron detector, which can reveal topological features via secondary electron (SE) imaging, while simultaneously capturing the thin NPG through its entire thickness (bulk) with transmitted electrons through bright field (BF) and dark field (DF) imaging. The chemical change in the NPG was recorded using a Bruker SDD EDS detector during in situ heating and post-heating experiments.

We observed Ostwald ripening during heating; where large pores grew larger at the expense of smaller ones. While densification was not observed under $800{ }^{\circ} \mathrm{C}$ as in previous studies [2], it was clearly observed as the surface area/volume ratio continued to decrease (Figure 1). After about 94 mins of heating, it is clear from Figure 1 that the number of pores decreased considerably with an increase in average size. The largest pores observed on the surface show clear faceting and a decrease in curvature as shown in Figure 2 (b). Twin boundaries were resolved in areas which underwent densification. EDX mapping showed no significant changes to the uniformity of the elemental distribution after heating (Figure 2-a).

In this study, the main aspects of morphological change during heating were captured using different characterization methods. Ostwald ripening slowed down considerably following the formation of surface facets. Faceting and twin formation were observed in large pores and densified areas respectively. Future work includes comparing this coarsening process with that for other compositions and in different gaseous environments [5]. 


\section{References:}

[1] Adrian Vega and Roger C. Newman, NSTI-Nanotech Conference proceedings (2012), p. 510.

[2] Yu-chen Karen Chen-Wiegart, Steve Wang, and Yong S. Chu, Acta Materialia 60 (2012), p. 4972.

[3] J. Erlebacher, Physical Review Letters 106 (2011), p. 225504.

[4] Josh Snyder, Piyapong Asanithi, Alan B. Dalton and Jonah Erlebacher, Advanced Materials 20 (2008), p. 4883.

[5] Electron microscopic studies were carried out at the Ontario Centre for the Characterisation of Advanced Materials (OCCAM), University of Toronto.
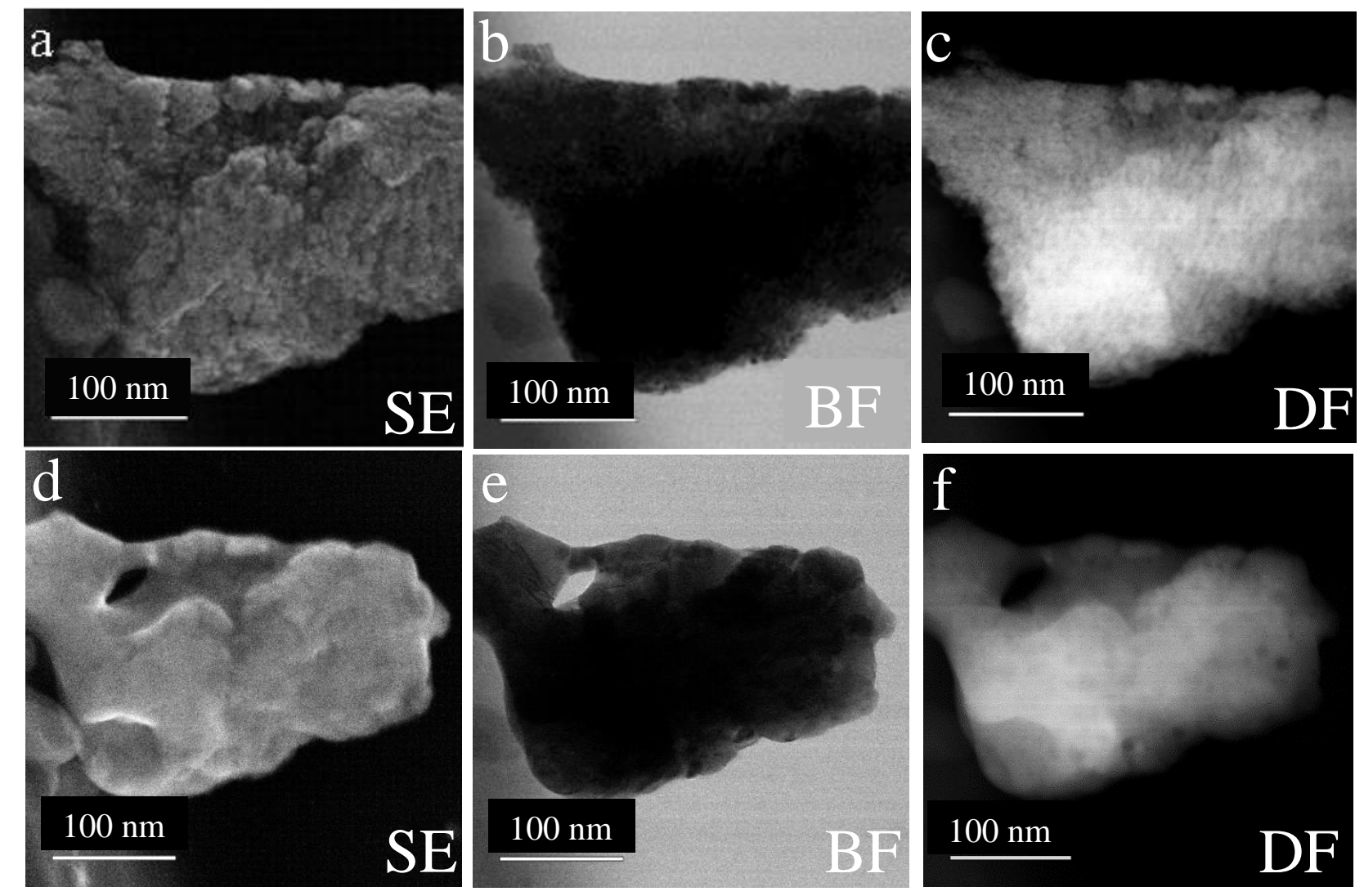

Figure 1. TEM/STEM images before heating (a-c) and after heating (d-f) for 94 mins at $440^{\circ} \mathrm{C}$.
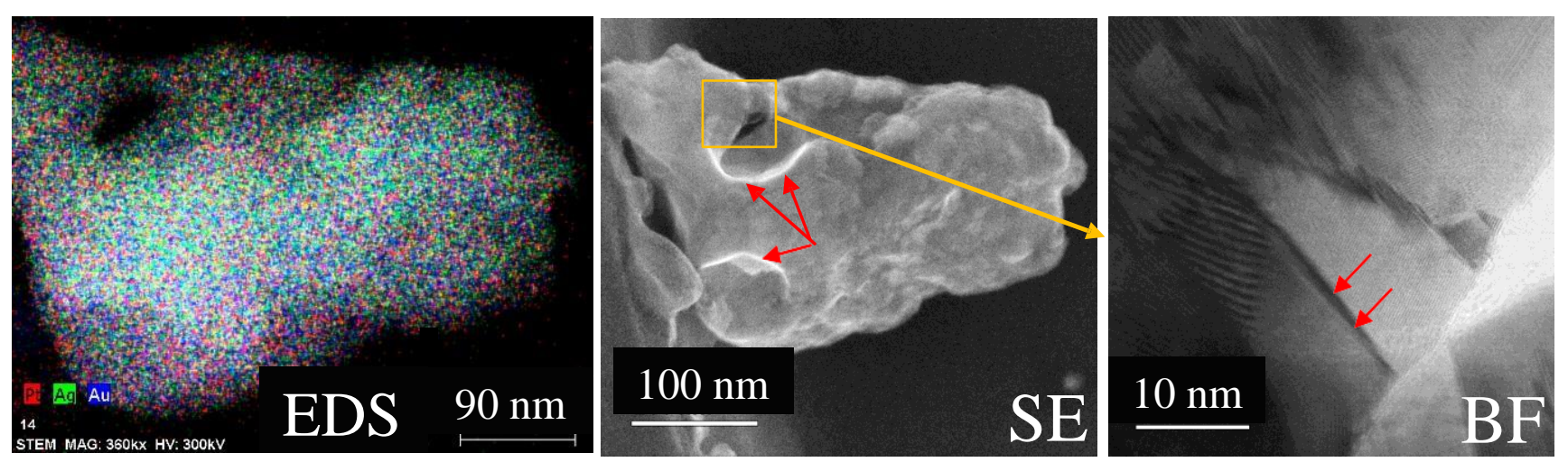

Figure 2. EDX map after heating (a) and prevalent morphological features at 48 mins of heating: (b) SE image showing faceting and (c) TEM image showing formed twin boundaries. 